\title{
Research Paper: Effect of Mindfulness-Based Therapy on Pain and Depression in Multiple Sclerosis Patients
}

Azadeh Choobforoushzadeh ${ }^{*}$ iD, Azra Mohammadpanah Ardakan ${ }^{1}$ (D)

1. Department of Psychology, Faculty of Humanistic Sciences, and Social Sciences, Ardakan University, Ardakan, Iran.

\begin{tabular}{|c|c|}
\hline & Chat on Choobforoushzadeh A \& Mohammadnanah Ardakan A (2019) Effect of Mindfulness-Based Therany on Pain and \\
\hline 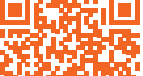 & $\begin{array}{l}\text { Depression in Multiple Sclerosis Patients. Journal of Practice in Clinical Psychology, 7(4), 271-280. http://dx.doi.org/10.32598/ } \\
\text { jpcp.7.4.271 }\end{array}$ \\
\hline Dingus & dol'http://dx.doi.org/10.32598/jpcp.7.4.271 \\
\hline
\end{tabular}

\section{(c) (i) (\$)}

Article info:

Received: 17 Feb 2019

Accepted: 10 Aug 2019

Available Online: 01 Oct 2019

\section{Keywords:}

Mindfulness-based therapy, Depression, Hospital anxiety and depression scale, McGill pain questionnaire, Multiple sclerosis, Pain

\section{ABSTRACT}

Objective: The frequency and impact of pain and depression have been long underestimated in Multiple Sclerosis (MS) patients. Therefore, systematic screening and management of pain and depression are recommended for these patients. The current study evaluated the effectiveness of Mindfulness-Based Therapy (MBT) in reducing pain and depression in patients with MS.

Methods: This was a quasi-experimental study with a pre-test, post-test and a control group design. We conducted a randomized trial on 24 MS patients with primary pain and depression. Moreover, they were members of the MS Society at Shahid Sadooghi Hospital in Yazd City, Iran, in 2017. The study participants were randomly divided into the test $(n=12)$ and control $(n=12)$ groups. The study participants were evaluated at 3-time points (baseline, end of the treatment, follow-up) using the McGill Pain Questionnaire and Depression subscale of the Hospital Anxiety and Depression Scale (HADS) as outcome measures.

Results: The repeated measures Analyses of Variance (ANOVA) results revealed that MBT significantly reduced pain and depression in MS patients compared to the controls $(\mathrm{P}<0.05)$. The effect of outcome measures was maintained at 2-months follow-up $(\mathrm{P}<0.05)$. The effect size on pain and depression were 0.41 and 0.43 , respectively.

Conclusion: MBT has been effective on pain and depression reduction among MS patients

\section{* Corresponding Author:}

Azadeh Choobforoushzadeh, PhD.

Address: Department of Psychology, Humanistic Sciences and Social Sciences, Ardakan University, Ardakan, Iran

Tel: +98 (35) 32240991

E-mail: azadechoobforoush@ardakan.ac.ir 


\section{Highlights}

- The problems associated with MS significantly affect the patients' psychological well-being.

- The frequency and impact of pain and depression have been long neglected in MS patients.

- MBT can reduce pain and depression in patients with MS.

\section{Plain Language Summary}

The frequency and impact of pain and depression have been long neglected in Multiple Sclerosis (MS) patients. Therefore, systematic screening and management of pain and depression are recommended for these patients. The current study evaluated the effectiveness of a Mindfulness-Based Therapy (MBT) program in reducing pain and depression in patients with MS. The results revealed that MBT program could significantly reduce pain and depression in these patients compared to the controls.

\section{Introduction}

ultiple Sclerosis (MS) is among the M common diseases of the autoimmune system (Pahlavanzadeh, Dalvi-Isfahani, Alimohammadi, \& Chitsaz, 2015). In other words, MS is an inflammatory disease of the Central Nervous System (CNS), which affects women and men in a ratio of 3:2. Moreover, it often develops in young people aged between 20 and 40 years. The generally accepted hypothesis for its etiology and pathogenesis is that this disease results from the activation of the immune system by one or more viruses in a genetically predisposed individual (Sevene et al. 2009).

MS is quintessentially a progressive disabling neurological disease. However, patients' experience of their disease extends beyond neurological disability to many other aspects of suffering, notably, symptoms of fatigue, cognitive impairment, depression, and pain (Siegert \& Abernethy, 2006).

Depression in people with chronic physical illnesses may exacerbate functional disability and lead to the increased use of healthcare services (Khazaeili, Zargham Hajebi, Mohamadkhani, \& Mirzahoseini, 2019) and reduced quality of life (Rickards 2005). Despite the high prevalence of these disorders, depression remains under-diagnosed and inadequately treated (Marrie et al. 2009; Sollom \& Kneebon, 2007). Major depression occurs in MS three times higher than the prevalence rate reported for psychiatric co-morbidity in community-based samples; it also exceeds that for other disabling neurological disorders. The literature highlights that depression is reported at a rate of $27 \%-54 \%$ in MS patients (McGuigan \& Hutchinson, 2006).
Various mechanisms may underlie the high prevalence of depression in these patients. Firstly, this may be understood as reactive stress and depression following the diagnosis and uncertainties about prognosis and the future in general. In the absence of adequate social support and the implementation of inappropriate coping strategies, this issue can reinforce and perpetuate the stressful reaction. If the patient fails to possess the necessary psychological resources to break this vicious circle, chronic clinical depression can develop over time. Secondly, the neuroinflammatory disease process in MS may contribute to the development or aggravation of depression. The release of pro-inflammatory cytokines, such as interferon-g, TNFan, or interleukin-6, may generate symptoms that reinforce an underlying tendency for depression, including the loss of appetite, sleep disturbances, asthenia, weight loss.

Moreover, biopsychological factors may interact to exacerbate depressive symptoms. For example, stress leads to the activation of the hypothalamus-pituitary-adrenal axis and of the sympathetic nervous system; in turn, it can, under the particular circumstance,s stimulate the immune system to release pro-inflammatory cytokines (Ziemssen, 2009). In other words, the complex interactions between brain pathology and environmental factors may determine psychological and psychiatric symptoms in MS patients (Khazaeili et al., 2019).

Pain is a frequent problem in MS patients (Nurmikko, Gupta, \& Maclver 2010). Furthermore, it is a complex symptom experienced in various manners. Pain prevalence (any type) among people with MS ranges widely, from $29 \%$ to $86 \%$. Pain is defined as "an unpleasant sensory and emotional experience associated with actual or potential tissue damage or described in terms of such 
damage" by the International Association for the Study of Pain (IASP). (Merskey, 2007). Therefore, pain has a biopsychological component. While pain is usually not an initial symptom of MS, the disease may bring about an acute pain experience, such as painful tonic spasms or Lhermitte's sign. In other patients, pain may become chronic as a long-term result of damage to nerve root entry zones (trigeminal neuralgia) or structures in central sensory pathways (Nurmikko, Gupta, \& Maclver 2010). However, medical intervention is frequently unable to resolve chronic pain, resulting in an increased need for pain management approaches, like other chronic conditions (Gatchel, 2004). Pain in patients with MS derives from a physical disorder of the CNS (neuropathic pain due to lesions along the sensory pathway that can cause burning or shock-like pain) and the musculoskeletal system (pain likely due to the movement impairment, e.g. back pain) (Saverino \& Solaro, 2012).

The strongest predictors of pain severity in MS are the presence of multiple pain sites, depression, higher disability level, and pain that interferes with daily living activities and enjoyment (Hadjimichael, Kerns, Rizzo, Cutter, \& Vollmer 2007). Pain in people with MS adversely affects most aspects of their life quality and significantly interferes with emotional and physical functioning, including sleeping, ability to work, maintaining relationships, and enjoying life (Saverino \& Solaro, 2012).

For people with MS, rehabilitation, with a focus on biopsychological purposes, impacts the quality of life and daily living activities. (Williams, Eccleston, \& Morley, 2012). Many people with MS report dissatisfaction with their pain management plans, and there is a compelling need to identify effective interventions that reduce the experience of pain (Ehde et al., 2015).

Recently, there has been increased attention to the psychological flexibility model; it extends the fear-avoidance model of chronic pain and proposes to improve treatment outcomes through the fostering of accepting attitudes towards pain (Pincus \& McCracken, 2013). Pain acceptance has demonstrated positive associations with cognitive, emotional, social, and occupational functioning as well as decreased pain catastrophizing in chronic pain populations (McCracken \& Eccleston, 2005). Pain acceptance is a particularly salient target for intervention in mindfulness- and acceptance-based therapies for chronic pain (Sturgeon, 2014).

Mindfulness meditation has a longstanding history in eastern practices and has recently received considerable public interest. Indeed, the science, practice, and implementation of Mindfulness-Based Interventions (MBIs) have dramatically increased in recent years. Mindfulness is a natural human state in which an individual experiences and attends to the present moment. Interventions have been developed to train individuals to incorporate this practice into daily life (Shapero, Greenberg, Pedrelli, de Jong, \& Desbordes 2018; Creswell, 2017).

MBIs, including Mindfulness-Based Stress Reduction (MBSR) and Mindfulness-Based Cognitive Therapy (MBCT), were developed as secular manualized groupbased intervention programs. These programs generally consist of 8 weekly 2 - to 2.5 -hour classes with approximately 12 patients. Additionally, these programs often include 1-day retreatment. A key feature of MBIs is the education in formal and informal mindfulness meditation practices to train the attentional control component as well as the non-judgmental attitudinal aspects of mindfulness as described above (Alsubaie et al., 2017; Crane et al., 2017).

Regular meditation practices include sitting meditation, mindful movement (including walking medication and gentle yoga exercises) and body scan, mindfully exploring bodily sensations, starting with the feet and progressively moving to the head and neck. The mindfulness meditation exercises focus on paying attention to bodily sensations, emotions, and thoughts while embracing a non-judgmental, accepting attitude towards whatever arises until it passes away (Stahl and Goldstein, 2010). MBIs have a significant homework component with guided (often with audio recording) and unguided meditation practices, i.e. assigned as daily home practice. The programs also include informal practices aimed to integrate mindful awareness in everyday activities, like mindful eating ("the raisin exercise") and mindful teeth brushing (Shapero, Greenberg, Pedrelli, de Jong, \& Desbordes, 2018).

MBIs are increasingly applied in healthcare settings around the world, in particular, as a means of managing stress in chronic conditions (Goyal et al., 2014). In other words, Mindfulness-Based Therapy (MBT) is a stress relief technique that reduces the cognitive function as well as the burden of symptoms (Frontario, Feld, Sherman, Krupp, \& Charvet 2016).

Numerous research investigations have suggested that MBIs are efficacious for reducing depression relapse and treating depression symptoms. Research has also provided evidence for the mechanisms of MBIs in improving psychological health. MBIs have increased positive psychological attributes, such as mindfulness, meta-awareness, and self-compassion. Besides, MBIs have been reported to reduce negative thinking patterns and reactions associated with psychopathologies, such as rumination, worry, and emotional reactivity. Further- 
more, MBIs could reduce cognitive deficits associated with psychiatric disorders, such as over-general autobiographical memory, poor attention regulation, and cognitive rigidity. Mindfulness meditation may also be associated with neural changes (Shapero et al., 2018).

Psychological problems in MS patients significantly affect the mental health of them and their caregivers. Thus, it is necessary to study the relevant internal and external impacting factors in the context of mindfulnessbased cognitive therapy. In addition, this study combined the techniques of MBSR and MBCT under the general title of MBT for the first time. Moreover, despite numerous studies available, there were no controlled studies about the effectiveness of MBT on pain and depression in MS patients. Therefore, the present study aimed to evaluate the effectiveness of MBT in the reduction of pain and depression in MS patients.

\section{Methods}

This was a quasi-experimental study with a pre-test, post-test and a control group design. The study was conducted at Shahid Sadooghi Hospital of the Yazd Medical School, in Yazd City, Iran, from April to July of 2017. The institutional review board approved the study protocol.

For sampling, first, the documents and an introduction letter were presented to the director and research department of the MS Society in Yazd Shahid Sadooghi Hospital. Then, the researchers participated in one of the patients' rounds, and a form, containing questions about demographic information, anxiety, and depression records, and study inclusion criteria were provided to them to complete. The ideal sample size in experimental studies is 12 individuals (Biyabangard, 2011); thus, 12 eligible patients were selected from Yazd $(\mathrm{N}=24)$.

Therefore, 24 participants with relapsing-remitting MS, followed as outpatients at the MS Society (at Shahid Sadooghi Hospital), were voluntarily recruited for the study. All study participants were clinically diagnosed by the McGuigan and Hutchinson (2006) criteria. They were in a stable phase of the disease, without relapsing in the last 2 months. The inclusion criteria were being $>18$-year-old, providing consent for participation in the study, being clinically diagnosed by the McGuigan and Hutchinson (2006) criteria, and experience primary pain and depression. The exclusion criteria were the acute relapse of MS within the last month, and the presence of additional neurological or psychiatric disorders, epilepsy, and other chronic diseases.
Written informed consent was obtained from all study participants, following a thorough description of the study procedures and requirements. Next, they were randomly assigned into two groups, each containing 12 patients. Participants assigned to the MBT group received 8 weeks of MBT (based on MBSR and MBCT). The course was delivered in 3-hour sessions, with time for breaks. The core components of MBSR are as follows: Mindful breath awareness, usually performed in a sitting position; mindful body awareness, usually conducted in a lying position, and mindful movement, usually involving simple yoga postures.

The core component of MBCT emphasizes on negative thought patterns in depression and the particular warning signs and triggers for depression. The study participants were encouraged to complete homework assignments to practice the learned techniques in the sessions. The remaining participants continued to receive Treatment As Usual (TAU) and constituted the control group. At the baseline visit, primary demographic data and disease history information were recorded and a complete neurological examination was performed; it included the assessment of neurological impairment using the Expanded Disability Status Scale (EDSS). Table 1 presents the general characteristics of the study participants.

The EDDS was used to rate the disability of the study participants at the study onset. Instruments used for the evaluation of intervention outcomes included the McGill Pain Questionnaire (MPQ) and the Hospital Anxiety and Depression Scale (HADS).

The EDDS is the oldest and the most widely used rating system of clinical assessment in MS (Sharrack and Hughes 1996). The EDSS is rated by half-point increments, from 0.0 (regular neurological examination) to 10.0 (death due to MS complications). Following the neurological examination, the investigator is required to summarize the results in several "functional system scores", which are graded from average $(0)$ to maximal impairment (5 or 6). The functional systems include the following systems: pyramidal, cerebellar, brain stem, sensory, bowel and bladder, visual, cerebral, and "others". An overall score for the patient's disability is then obtained by combining the different functional system grades and the ability to walk; it has to be assessed separately, to provide a score on the full 20-point scale. EDSS steps 1.0 to 4.5 refer to people with MS who are entirely ambulatory. EDSS steps 5.0 to 9.5 are defined by the impairment of ambulation (Hauser \& Goodkin, 2001). 
MPQ primarily consists of 3 significant classes of word descriptors; sensory, affective, and evaluative, i.e. used by patients to specify subjective pain experience. It also contains an intensity scale and other items to determine the characteristics of the pain experience. The questionnaire was designed to provide quantitative measures of clinical pain that can be treated statistically (Ebrahimi-Nejad, Ebrahimi-Nejad, Kohan, \& Bahrampour-Nejad 2004).

The study participants also completed the HADS for the assessment of depressive symptoms. The HADS is a self-report 14-question survey (Bjelland, Dahl, Haug, \& Neckelmann 2002) used to identify anxiety and depression. The questionnaire has 7 questions reflecting anxiety alternating with 7 reflecting depression: HADS-A and HADS-D. Each item is answered on a 4-point (03 ) response category; the possible scores range from 0 to 21 for anxiety and depression. Several questions are reversed scored. A score of 0 to 7 for either subscale is regarded as the normal range, a score of 8 to 10 suggests mild anxiety or depression, a score of 11-14 reflects a moderate degree of anxiety or depression, and scores $\geq 15$ indicate more severe anxiety or depression. If participants obtained scores of anxiety or depression above 14 , the clinical nurse specialist at the relevant hospital was contacted and requested to follow-up the case.

SPSS was used to analyze the collected data using descriptive statistics, such as mean and standard deviation scores. The assessment took place before the treatment (T0), immediately after the treatment (T1), and at a twomonth follow-up (T2).

\section{Results}

We first conducted descriptive statistics, including an examination of the distributions for all continuous variables. Two repeated measures Analyses of Variance (ANOVA) (treatment condition $\mathrm{X}$ assessment period) were next conducted to examine the effect of MBT on depression and pain in MS patients, respectively. Effect sizes were computed by Cohen's $d$.

The Mean \pm SD age of the study patients was similar in the MBT and control groups (34.12 \pm 7.21 and $33.39 \pm 7.72$, respectively; $\mathrm{P}=0.678$ ). T-test results indicated no difference between the intervention and control groups concerning disease duration (mean $\pm \mathrm{SD}$ : $6.54 \pm 4.52$ and $6.24 \pm 4.36$, respectively; $\mathrm{P}=0.598$ ). Additionally, there were no significant differences in gender or EDSS between the two groups. The mean \pm SD scores of both dependent variables at pre-test (T0),post-test (T1), and follow-up stages (T2) are presented in Table
2. The assumption of normality was approved by the Shapiro-Wilk test. Moreover, the equality of population variances was approved by Levene's test. The two study groups did not significantly differ at pre-test in terms of pain and depression $(\mathrm{t}(22)=0.18, \mathrm{P}>.0 .05$, and $\mathrm{t}(22)$ $=0.20, \mathrm{P}>0.05$, for both measurements, respectively).

The 2 (group: MBT vs. control) $\times 3$ (time: Pre-test vs.post-test vs. follow-up) repeated measures ANOVA, with pain scores as the outcome variable, revealed a similar pattern; there was a significant main effect of time $(\mathrm{F} 2,21=7.3 ; \mathrm{P}<0.01)$ and a significant interaction effect of group and time $(F 2,21=10.1 ; \mathrm{P}<0.01)$. The obtained mean scores of pain indicated a significant improvement in the MBT group members; however, control individuals remained unchanged in this regard. Furthermore, the most significant improvement on pain scores was observed between pre-test and post-test; a t-test with pain change score ( post-test minus pre-test) as the dependent variable was significant $[\mathrm{t}(22)=-2.37, \mathrm{P}<0.001$; mean change scores: -6.7 and 1.13 for the MBI and control groups, respectively]. Between post-test and follow-up, the scores of both groups remained stable; i.e. changes in scores from $\mathrm{T} 1$ to $\mathrm{T} 2$ were not significantly different in both groups $[\mathrm{t}(22)=.5, \mathrm{P}>0.05$, mean changes scores: 0.11 and .05 , for the MBT and control groups, respectively], indicating that the MBT group's pain score improvement persisted at follow-up; whereas the control groups' unchanged higher pain scores remained the same.

The 2 (group: MBT vs. waiting) $\times 3$ (time: Pre-test vs post-test vs. follow-up) repeated measures ANOVA with depression as the outcome variable revealed a significant main effect of time $\left(\mathrm{F}_{2,21}=6.11 ; \mathrm{P}<.005\right)$ and a significant interaction effect of group and time $\left(\mathrm{F}_{2,21}=11.7 ; \mathrm{P}<.005\right)$. The achieved mean scores indicated that depression improved in the treatment group; however, the controls remained stable across all time points. The most significant improvement on depression scores was observed between T0 and T1; an Independent Samples t-test with depression change score ( post-test minus pre-test) as the dependent variable was significant $[\mathrm{t}(22)=-2.58$, $\mathrm{P}<0.001$; mean change scores: -4.42 and 0.7 for the MBT and control groups, respectively]. Between T1 and T2, the scores of both groups remained stable; i.e. changes in scores from $\mathrm{T} 1$ to $\mathrm{T} 2$ were not significantly different for both groups $[\mathrm{t}(22)=0.74, \mathrm{P}>0.05$; mean change scores: 0.08 and 0.28 for the MBT and control groups, respectively], indicating that the MBT group's improvement persisted at follow-up; however, the control groups' relatively unchanged higher depression scores persisted. 
Table 1. General characteristics of the study participants $(n=12)$

\begin{tabular}{ccc} 
Characteristic & MBT Group & TAU Group \\
\hline Age $(\mathrm{y})$ & $34.12 \pm 7.21$ & $33.39 \pm 7.72$ \\
\hline Gender (male/female) & $6 / 6$ & $6 / 6$ \\
Disease duration (y) & $6.54 \pm 4.52$ & $6.24 \pm 4.3$ \\
EDSS & $3.7 \pm 1.5$ & $3.9 \pm 1.4$ \\
\hline
\end{tabular}

Values are Mean $\pm S D$ for age, disease duration, and EDSS

Table2. Pain and depression scores in the MBT and TAU groups

\begin{tabular}{|c|c|c|c|c|}
\hline \multicolumn{5}{|c|}{ Mean $\pm S D$} \\
\hline & \multicolumn{2}{|c|}{ Depression } & \multicolumn{2}{|c|}{ Pain } \\
\hline & MBT & TAU & MBT & TAU \\
\hline TO & $15.52 \pm 2.39$ & $16.27 \pm 3.41$ & $18.92 \pm 8.56$ & $19.90 \pm 10.64$ \\
\hline $\mathrm{T} 1$ & $10.10 \pm 3.03$ & $16.97 \pm 3.27$ & $12.85 \pm 8.03$ & $21.03 \pm 10.33$ \\
\hline $\mathrm{T} 2$ & $10.18 \pm 2.37$ & $16.69 \pm 3.33$ & $12.94 \pm 8.14$ & $21.08 \pm 10.42$ \\
\hline
\end{tabular}

The effect size of MBT in this study, compared to the TAU group, was as Cohen's $\mathrm{d}=0.41$ for pain and Cohen's $\mathrm{d}=0.43$ for depression. Overall, the analyses suggested that MBT significantly reduced pain and depression in the test group compared with the control group.

\section{Discussion}

The current study results demonstrated a significant difference in thepost-test and follow-up scores of pain and depression between the test and control groups. Therefore, MBT has reduced pain and depression in MS patients. Some of these findings were consistent with those of Simpson et al. and Arnet and colleagues. Depression can result from multiple effects associated with managing a chronic condition. The use of MBIs in the MS population has been neglected (Simpson et al., 2014). Simpson et al., (2014) published a systematic review of MBIs in MS; they referred to two studies that assessed the effect of MBIs on depression in MS patients. Grossman et al., (2010) used the Center for Epidemiological Studies Depression (CES-D) scale, reporting significant reductions in both the whole intervention group and subgroup analyses of those with pre-intervention impairment at intervention completion. This was maintained at 6-month follow-up in the overall group as well as subgroup analyses. Mills and Allen (2000) also reported a significant change using the POMS scale.
Tavee et al., (2011) investigated the effects of meditation on pain and quality of life in MS and poly-neuropathy; they described a significant reduction in bodily pain, measured by the Visual Analogue Scale (VAS).

Along with the above findings, there is level B evidence (probably effective) for the efficacy of mindfulness training on psychological distress, depression, anxiety, pain, and quality of life in patients with progressive MS. Most of the worries that generate depression and anxiety in patients with MS include the fear of acute illness, emotional tension and movement problems, and chronic pain. Psychiatric interventions improve lethargy and depression in this population (Venasse, Edwards, \& Pilutti 2018).

MBT is based on the concepts of mental training that propose the nonjudgmental awareness of moment-tomoment experience (i.e. mindfulness) may positively affect the accuracy of perception, acceptance of intractable health-related changes, realistic sense of control, and appreciation of life experiences (Kabat-Zinn, 2009).

Similar to psychological acceptance, which fosters a nonjudgmental approach to distressing thoughts and emotions, pain acceptance is defined as a process of nonjudgmentally acknowledging pain, stopping maladaptive attempts to control pain, and learning to live a more productive life despite pain (McCracken \& Eccleston, 2005). Furthermore, the study findings revealed 
that the relationship between pain and mindfulness was strong and clinically significant $(\mathrm{t}=-5.52, \mathrm{P}<.0001)$. For each 18-point increase in mindfulness scores, pain interference scores are expected to decrease by 3.96 points $(95 \% \mathrm{CI}=-2.52$ to -5.40$)(\beta=-0.22, \mathrm{P}<.0001)$ (Senders et al. 2018). Pain acceptance influences emotional functioning through two distinct mechanisms; willingness to experience pain, which buffers against negative emotional reactions to pain, and continued engagement in valued activities despite the presence of pain, which bolsters positive emotions (Kranz, Bollinger, \& Nilges 2010). Acceptance of pain is theorized to uncouple the occurrence of catastrophic thoughts about pain from subsequent emotional suffering (Vowles, McCracken, \& Eccleston2008). It also reduces reliance on control- or avoidance-based coping strategies (McCracken and Eccleston, 2005); thereby, freeing cognitive and emotional resources for more meaningful pursuits (Sturgeon, 2014).

Frewen, Evans, Maraj, Dozois, \& Partridge (2008) focused on people's reactions to their negative thoughts. They assessed the occurrence frequency of negative thoughts and individuals' ability to 'let go' of them. They assessed mindfulness using the Mindful Attention Awareness Scale (MAAS; Brown and Ryan 2003), the Kentucky Inventory of Mindfulness Skills (KIMS; Baer, Smith, \& Allen 2004), and a behavioral test of breath focus versus mind wandering over a 15 -min sitting with instructions to focus on the breath. They found that naturally occurring variations in trait mindfulness correlated with both frequency and the ability to let go of negative thoughts. In a second study conducted in students referring to a student-counseling service, MAAS and KIMS changed from pre- to post-treatment in those participating in mindful awareness training (based on MBSR and MBCT). As predicted, frequency and letting go changed, as well. This study was an essential first test, using a correlational design (at one-time point and changes across time) demonstrating that certain key variables are associated with mindfulness. The ability to let go of thoughts is the key to coping with the tendency to consider one's mind, its contents and processes, as uncontrollable (Williams, Eccleston, \& Morley 2012).

MBT might be associated with a general reduction in stress, perhaps by encouraging patients to relate differently to their physical symptoms, so that when they occur, their consequences are less disturbing. Depression could be reduced by training patients on the awareness of negative thinking patterns reactivated during dysphoria and disengage from those ruminative depressive cycles. MBCT was designed to achieve these aims (Segal, Williams, \& Teasdaleet 2002).
The importance of continued research in this field is illustrated by studies suggesting that depression (Chwastiak \& Ehde, 2007) and pain appear to be undertreated in MS outpatient settings. Moreover, whereas clinicians are more concerned about the physical manifestation of the disease, patients with MS consider mental health to be a critical determinant of overall burden (Nordin \& Rorsman, 2012). Further focus on empirically-proven psychotherapeutic interventions may help bridge this gap and improve the overall quality of life in patients with MS.

MBT has been effective on pain and depression among MS patients. In sum, our findings are promising and support the use of MBT for pain and depression in the MS population. This pattern of results suggests that MBT may not be diagnosis-specific; however, instead, may address processes that occur in multiple disorders by changing a range of emotional and evaluative dimensions that underlie general aspects of well-being. Therefore, MBT may have general applicability and can be useful for patients with MS in improving their health in completing medical treatments. A limitation to this study was the lack of a follow-up period of more than several months, because of time constraints. Thus, the study outcomes should only be generalized to patients with relapsing-remitting MS. Further controlled studies, employing larger sample sizes with more extended follow-up stages, are necessary. Moreover, it is suggested that a more extended follow-up period be used to evaluate the continuation of treatment outcomes in future studies. Another suggestion is that, considering the effectiveness of MBT, use this educational package to improve anxiety and other psychological conditions. It is also recommended that the MS Association use this training package.

\section{Ethical Considerations}

\section{Compliance with ethical guidelines}

All ethical principles were met in this study. All procedures were according to the ethical standards of the responsible committee on human experimentation (national and institutional). The study participants were informed of the research objective and methods and informed consent was obtained from them. They were also assured of the confidentiality of their information. Moreover, they were free to leave the study at any time, and if desired, the results of the research would be available to them. 


\section{Funding}

This research received no specific grant from any funding agency in the public, commercial, or not-for-profit sectors.

\section{Authors' contributions}

All authors contributed equally in preparing this article.

\section{Conflict of interest}

The authors declare no conflicts of interest.

\section{Acknowledgments}

The authors would like to sincerely thank the members of the MS Society at Shahid Sadooghi Hospital in Yazd City for their cooperation.

\section{References}

Alsubaie, M., Abbott, R., Dunn, B., Dickens, C., Keil, T. F., \& Henley, W., et al. (2017). Mechanisms of action in Mindfulness-Based Cognitive Therapy (MBCT) and MindfulnessBased Stress Reduction (MBSR) in people with physical and/ or psychological conditions: A systematic review. Clinical Psychology Review, 55, 74-91. [DOI:10.1016/j.cpr.2017.04.008] [PMID]

Arnett, P. A., Barwick, F. H., \& Beeney, J. E. (2008). Depression in multiple sclerosis: Review and theoretical proposal. Journal of the International Neuropsychological Society, 14(5), 691-724. [DOI:10.1017/S1355617708081174] [PMID]

Baer, R. A., Smith, G. T., \& Allen, K. B. (2004). Assessment of mindfulness by self-report: The Kentucky inventory of mindfulness skills. Assessment, 11(3), 191-206. [DOI:10.1177/1073191104268029] [PMID]

Biyabangard, S. (2011). [The methods of research in psychology and educational science (Persian)]. Tehran: Doran.

Bjelland, I., Dahl, A. A., Haug, T. T., \& Neckelmann, D. (2002) The validity of the Hospital Anxiety and Depression Scale: An updated review. Journal of Psychiatric Research, 52(2), 6977. [DOI:10.1016/S0022-3999(01)00296-3]

Brown, K. W., \& Ryan, R. M. (2003). The benefits of being present: Mindfulness and its role in psychological well-being. Journal of Personality and Social Psychology, 84(4), 822-48. [DOI:10.1037/0022-3514.84.4.822] [PMID]

Burckhardt, C. S., \& Jones, K. D. (2003). Adult measures of pain The McGill Pain Questionnaire (MPQ), Rheumatoid Arthritis Pain Scale (RAPS), Short-Form McGill Pain Questionnaire (SF-MPQ), Verbal Descriptive Scale (VDS), Visual Analog Scale (VAS), and West Haven-Yale Multidisciplinary Pain Inventory (WHYMPI). Arthritis Care \& Research, 49(S5), S96S104. [DOI:10.1002/art.11440]
Chwastiak, L. A., \& Ehde, D. M. (2007). Psychiatric issues in multiple sclerosis. The Psychiatric Clinics of North America, 30(4), 803-17. [DOI:10.1016/j.psc.2007.07.003] [PMID] [PMCID]

Crane, R. S., Brewer, J., Feldman, C., Kabat-Zinn, J., Santorelli, S., \& Williams, J. M., et al. (2017). What defines mindfulnessbased programs? The warp and the weft. Psychological Medicine, 47(6), 990-9. [DOI:10.1017/S0033291716003317] [PMID]

Creswell, J. D. (2017). Mindfulness interventions. Annual Review of Psychology, 68, 491-516. [DOI:10.1146/annurevpsych-042716-051139] [PMID]

Ebrahimi-Nejad, G. R., Ebrahimi-Nejad, A., Kohan, S., \& Bahrampour, A. (2004). [The evaluation of pain in neurosurgical patients before and after operation in Kerman Shahid Bahonar Hospital according to the McGill Pain Questionnaire (Persian)]. Journal of Kerman University of Medical Sciences, 11(2), $119-25$

Ehde, D. M., Alschuler, K. N., Osborne, T. L., Hanley, M. A., Jensen, M. P., Kraft, G. H. (2015). Utilization and patients' perceptions of the effectiveness of pain treatments in multiple sclerosis: A cross-sectional survey. Disability and Health Journal, 8(3), 452-6. [DOI:10.1016/j.dhjo.2015.03.001] [PMID] [PMCID]

Frewen, P. A., Evans, E. M., Maraj, N., Dozois, D. J. A., \& Partridge, K. (2008). Letting go: Mindfulness and negative automatic thinking. Cognitive Therapy and Research, 32(6), 758-74 [DOI:10.1007/s10608-007-9142-1]

Frontario, A., Feld, E., Sherman, K., Krupp, L., \& Charvet, L. (2016). Telehealth mindfulness meditation improves cognitive performance in adults with Multiple Sclerosis (MS). Neurology, 86(16 Suppl), P3.092.

Gatchel, R. J. (2004). Comorbidity of chronic pain and mental health disorders: The biopsychosocial perspective. The American Psychologist, 59(8), 795-805. [DOI:10.1037/0003066X.59.8.795] [PMID]

Goyal, M., Singh, S., Sibinga, E. M., Gould, N. F., RowlandSeymour, A., \& Sharma, R., et al. (2014). Meditation programs for psychological stress and wellbeing: A systematic review and meta-analysis. JAMA Internal Medicine; 174(3), 357-68. [DOI:10.1001/jamainternmed.2013.13018] [PMID] [PMCID]

Grossman, P., Kappos, L., Gensicke, H., D'Souza, M., Mohr, D. C., \& Penner, I. K., et al. (2010). MS quality of life, depression, and fatigue improve after mindfulness training: A randomized trial. Neurology, 75(13), 1141-9. [DOI:10.1212/ WNL.0b013e3181f4d80d] [PMID] [PMCID]

Hadjimichael, O., Kerns, R. D., Rizzo, M. A., Cutter, G., Vollmer, T. (2007). Persistent pain and uncomfortable sensations in persons with multiple sclerosis. Pain, 127(1-2), 35-41. [DOI:10.1016/j.pain.2006.07.015] [PMID]

Hauser, S. L., \& Goodkin, D. E. (2001). Multiple sclerosis and other demyelinating disease. In Braunwald E, Fauci AD Kasper DL, et al (eds): Harrison's principles of internal medicine. (pp. 2452-60). $15^{\text {th }}$ ed. New York: McGraw-Hill.

Hölzel, B. K., Lazar, S. W., Gard, T., Schuman-Olivier, Z., Vago, D. R., \& Ott, U. (2011). How does mindfulness meditation work? Proposing mechanisms of action from a conceptual and neural perspective. Perspectives on Psychological Science, 6(6), 537-59. [DOI:10.1177/1745691611419671] [PMID] 
Kabat-Zinn, J., \& Hanh, T. N. (2009). Full catastrophe living: Using the wisdom of your body and mind to face stress, pain, and illness. Delta. ISBN: 0307567575.

Khazaeili, M., Zargham Hajebi, M., Mohamadkhani, P., \& Mirzahoseini, H. (2019). The effectiveness of mindfulness-based intervention on anxiety, depression and burden of caregivers of multiple sclerosis patients through web conferencing. Practice in Clinical Psychology, 7(1), 21-32. [DOI:10.32598/ jpcp.7.1.21]

Kranz, D., Bollinger, A., \& Nilges, P. (2010). Chronic pain acceptance and affective well-being: A coping perspective. European Journal of Pain (London, England), 14(10), 1021-5. [DOI:10.1016/j.ejpain.2010.03.010] [PMID]

Marrie, R. A., Horowitz, R., Cutter, G., Tyry, T., Campagnolo, D., \& Vollmer, T. (2009). The burden of mental comorbidity in multiple sclerosis: Frequent, underdiagnosed and undertreated. Multiple Sclerosis, 15(3), 385-92. [DOI:10.1177/1352458508099477] [PMID]

McCracken, L. M., \& Eccleston, C. (2005). A prospective study of acceptance of pain and patient functioning with chronic pain. Pain, 118(1-2), 164-9. [DOI:10.1016/j.pain.2005.08.015] [PMID]

McCracken, L. M., Vowles, K. E., \& Eccleston, C. (2004). Acceptance of chronic pain: Component analysis and a revised assessment method. Pain, 107(1-2), 159-66. [DOI:10.1016/j. pain.2003.10.012] [PMID]

McGuigan, C., \& Hutchinson, M. (2006). Unrecognised symptoms of depression in a community-based population with multiple sclerosis. Journal of Neurology, 253(2), 219-23. [DOI:10.1007/s00415-005-0963-0] [PMID]

Merskey, H. (2007). The taxonomy of pain. The Medical Clinics of North America, 91(1), 13-20. [DOI:10.1016/j.mcna.2006.10.009]

Mills, N., \& Allen, J. (2000). Mindfulness of movement as a coping strategy in multiple sclerosis: A pilot study. General Hospital Psychiatry, 22(6), 425-31. [DOI:10.1016/S01638343(00)00100-6]

National Institute for Health and Clinical Excellence. (2015) Depression in adults: The treatment and management of depression in adults. Retrieved from: www.nice.org.uk/guidance/cg90.

Nordin, L., \& Rorsman, I. (2012). Cognitive behavioural therapy in multiple sclerosis: A randomized controlled pilot study of acceptance and commitment therapy. Journal of Rehabilitation Medicine, 44(1), 87-90. [DOI:10.2340/16501977-0898] [PMID]

Nurmikko, T. J., Gupta, S., \& Maclver, K. (2010). Multiple sclerosis-related central pain disorders. Current Pain and Headache Reports, 14(3), 189-95. [DOI:10.1007/s11916-010-0108-8] [PMID]

Pahlavanzadeh, S., Dalvi-Isfahani, F., Alimohammadi, N., \& Chitsaz, A. (2015). The effect of group psycho-education program on the burden of family caregivers with multiple sclerosis patients in Isfahan in 2013-2014. Iranian Journal of Nursing and Midwifery Research, 20(4), 420-5. [DOI:10.4103/1735-9066.161000] [PMID] [PMCID]

Pincus, T., \& McCracken, L. M. (2013). Psychological factors and treatment opportunities in low back pain. Best Practice $\mathcal{E}$ Research Clinical Rheumatology, 27(5), 625-35. [DOI:10.1016/j. berh.2013.09.010] [PMID]
Rickards, H. (2005). Depression in neurological disorders: Parkinson's disease, multiple sclerosis and stroke. Journal of Neurology, Neurosurgery \& Psychiatry, 76(Suppl 1), i48-i52. [DOI:10.1136/ jnnp.2004.060426] [PMID] [PMCID]

Saverino, A., \& Solaro, C. (2012). Pain in individuals with multiple sclerosis, knee prosthesis, and post-herpetic neuralgia: Learning from focus group patients' experience. The Clinical Journal of Pain, 28(4), 300-8. [DOI:10.1097/AJP.0b013e31823216b4] [PMID]

Segal, Z. V., Williams, J. M. G., \& Teasdale, J. D. (2002). Mindfulness-based cognitive therapy for depression: A new approach to preventing relapse. New York: Guilford Publications.

Senders, A., Borgatti, A., Hanes, D., \& Shinto, L. (2018). Association between pain and mindfulness in multiple sclerosis: A cross-sectional survey. International Journal of MS Care, 20(1), 28-34. [DOI:10.7224/1537-2073.2016-076] [PMID] [PMCID]

Sevène, A., Akrour, B., Galimard-Maisonneuve, E., Kutneh, M. Royer, P., \& Sevène, M. (2009). Multiple sclerosis and sexuality: A complex model. Sexologies, 18(2), 86-90. [DOI:10.1016/j. sexol.2009.01.011]

Shapero, B. G., Greenberg, J., Pedrelli, P., de Jong, M., \& Desbordes, G. (2018). Focus (American Psychiatric Association Publishing), 16(1), 32-9. [DOI:10.1176/appi.focus.20170039] [PMID] [PMCID]

Sharrack, B., \& Hughes, R. A. C. (1996). Clinical scales for multiple sclerosis. Journal of the Neurological Sciences, 135(1), 1-9. [https://doi.org/10.1016/0022-510X(95)00261-Y]

Siegert, R. J., \& Abernethy, D. A. (2005). Depression in multiple sclerosis: A review. Journal of Neurology Neurosurgery Psychiatry, 76(4), 469-75. [DOI:10.1136/jnnp.2004.054635] [PMID] [PMCID]

Simpson, R., Booth, J., Lawrence, M., Byrne, S., Mair, F., \& Mercer, S. (2014). Mindfulness based interventions in multiple sclerosis--a systematic review. BMC Neurology, 14, 15. [DOI:10.1186/1471-2377-14-15] [PMID] [PMCID]

Simpson, R., Mair, F., \& Mercer, S. (2015). Mindfulness-based interventions for people with multiple sclerosis. Multiple Sclerosis Journal, 21(9), 1093-4. [DOI:10.1177/1352458515579702] [PMID]

Sollom, A. C., \& Kneebone, I. I. (2007). Treatment of depression in people who have multiple sclerosis. Multiple Sclerosis Journal, 13(5), 632-5. [DOI:10.1177/1352458507072384] [PMID]

Stahl, B., \& Goldstein, E. (2010). A mindfulness-based stress reduction workbook. Oakland, CA: New Harbinger Publications.

Sturgeon, J. A. (2014). Psychological therapies for the management chronic pain. Psychology Research and Behavior Management, 7, 115-24. [DOI:10.2147/PRBM.S44762] [PMID] [PMCID]

Tavee, J., Rensel, M., Planchon, S. M., Butler, R. S., \& Stone, L. (2011). Effects of meditation on pain and quality of life in multiple sclerosis and polyneuropathy: A controlled study. International Journal of MS Care, 13(4), 163-8. [DOI:10.7224/1537-207313.4.163] [PMID] [PMCID]

Venasse, M., Edwards, T. \& Pilutti, L. (2018). Exploring wellness interventions in progressive multiple sclerosis: An evidencebased review. Current Treatment Options in Neurology, 20(5), 13 [DOI:10.1007/s11940-018-0497-2] [PMID] 
Vowles, K. E., McCracken, L. M., \& Eccleston, C. (2008). Patient functioning and catastrophizing in chronic pain: The mediating effects of acceptance. Health Psychology, 27(Suppl 2), S136-S43. [DOI:10.1037/0278-6133.27.2(Suppl.).S136]

Williams, A. C., Eccleston, C., \& Morley, S. (2012). Psychological therapies for the management of chronic pain (excluding headache) in adults. Cochrane Database of Systematic Reviews, 11, CD007407. [DOI:10.1002/14651858.CD007407.pub3] [PMID] [PMCID]

Ziemssen, T. (2009). Multiple sclerosis beyond EDSS: Depression and fatigue. Journal of the Neurological Sciences, 277(Suppl 1), S37-S41. [DOI:10.1016/S0022-510X(09)70011-5] 